ncov/vaccines/recommendations.html. Published 2020. Accessed February $8,2021$.

6. Oliver SE, Gargano JW, Marin M, et al. The Advisory Committee on Immunization Practices interim recommendation for use of PfizerBioNTech COVID-19 vaccine-United States, December 2020. Morb Mortal Wkly Rep 2020;69:1922-1924.
7. The Vaccines and Related Biological Products Advisory Committee meeting, December 10, 2020. FDA Briefing Document, Pfizer-BioNTech COVID-19 vaccine. US Food and Drug Administration website. https://www.fda.gov/ media/144245/download. Published 2020. Accessed February 8, 2021.

\title{
Impact of coronavirus disease 2019 (COVID-19) vaccination program on healthcare worker infections in an academic hospital
}

\author{
Erin Dunbar MD, MS, FAAP ${ }^{1}$ (1), Emily Godbout DO, $\mathrm{MPH}^{2}$, Rachel Pryor RN, MPH${ }^{3}$, Henry J. Rozycki MD ${ }^{4}$ and \\ Gonzalo Bearman MD, MPH${ }^{2}$ \\ ${ }^{1}$ Pediatric Emergency Department, Virginia Commonwealth University Health System, Richmond, Virginia, ${ }^{2}$ Department of Infectious Diseases, Virginia \\ Commonwealth University Health System, Richmond, Virginia, ${ }^{3}$ Hospital Infection Prevention Program, Virginia Commonwealth University Health System, \\ Richmond, Virginia and ${ }^{4}$ Department of Pediatrics, Division of Neonatal-Perinatal Medicine, Virginia Commonwealth University Health System, Richmond, Virginia
}

To the Editor-The third wave of the coronavirus disease 2019 (COVID-19) pandemic resulted in a significant rise in hospitalizations and healthcare worker (HCW) severe acute respiratory coronavirus virus 2 (SARS-CoV-2) infections. After implementing the SARS-CoV-2 vaccination program, despite rising COVID-19 hospitalizations, we promptly observed a decrease in HCW infections.

Our COVID-19 vaccination HCW program began on December 16, 2020, (Pfizer/BioNTech) and December 28, 2020 (Moderna). The COVID-19 cases were identified by nasal swab PCR testing of clinically symptomatic individuals. Six days after beginning employee immunizations, our HCW COVID-19 infection rate decreased by $25 \%$. After $60 \%$ of employees received the 1 st vaccine dose, the HCW COVID-19 rate decreased by $50 \%$ (Fig. 1). At 14-28 days and $>28$ days after their first vaccine dose, HCWs were less likely to have COVID-19 than those who did not receive the vaccine $(0.15 \%$ and $0.00 \%$ vs $0.59 \%, P=.0002$ and .0004 , respectively). Concurrently implemented SARS-CoV-2 transmission prevention strategies included the transition from cloth masks to level-3 masks for all employees, mandatory face shields for direct patient care, a restricted visitor policy, and physical space adjustments for improved social distancing.

The Pfizer/BioNTech clinical trial reported a vaccine efficacy of $95 \%$ at least 7 days after the second dose and protection as early as 12 days after administration of the first dose. ${ }^{2}$ The Moderna vaccine trial observed similar protection prior to the second dose. ${ }^{3}$ Our data are consistent with these studies and underscore the prompt benefits of vaccination for the prevention of COVID-19 in the healthcare system even prior to the completion of the second vaccine dose. Our additional infection control strategies are consistent with CDC recommendations ${ }^{4}$ and likely further optimized HCW safety.

We present a bundled infection prevention approach including vaccination for the prompt reduction of COVID-19 infection in HCWs. The impact of COVID-19 vaccination in HCWs was observed even prior to completion of the second dose. Wood et $\mathrm{al}^{5}$ suggest $12 \mathrm{key}$ strategies to promote vaccination, 2 of which are relevant here: increasing observability and countering anecdotal "bad reaction" with "good reaction" vaccine stories. ${ }^{5} \mathrm{We}$ share our vaccine story to encourage more vaccination-hesitant HCWs to receive immunizations and to receive them earlier. Reaching herd immunity through vaccination is a crucial next step in ending this pandemic.

Acknowledgments.

Financial support. No financial support was provided relevant to this article.

Conflicts of interest. All authors report no conflicts of interest relevant to this article.

\section{References}

1. Coronavirus key measures-COVID 19 in Virginia. Virginia Department of Health website. https://www.vdh.virginia.gov/coronavirus/key-measures/. Published 2020. Accessed February 8, 2021.
Author for correspondence: Erin Dunbar, E-mail: erin.dunbar@vcuhealth.org

Cite this article: Dunbar E, et al. (2022). Impact of coronavirus disease 2019 (COVID-

19) vaccination program on healthcare worker infections in an academic hospital. Infection

Control \& Hospital Epidemiology, 43: 680-681, https://doi.org/10.1017/ice.2021.62 


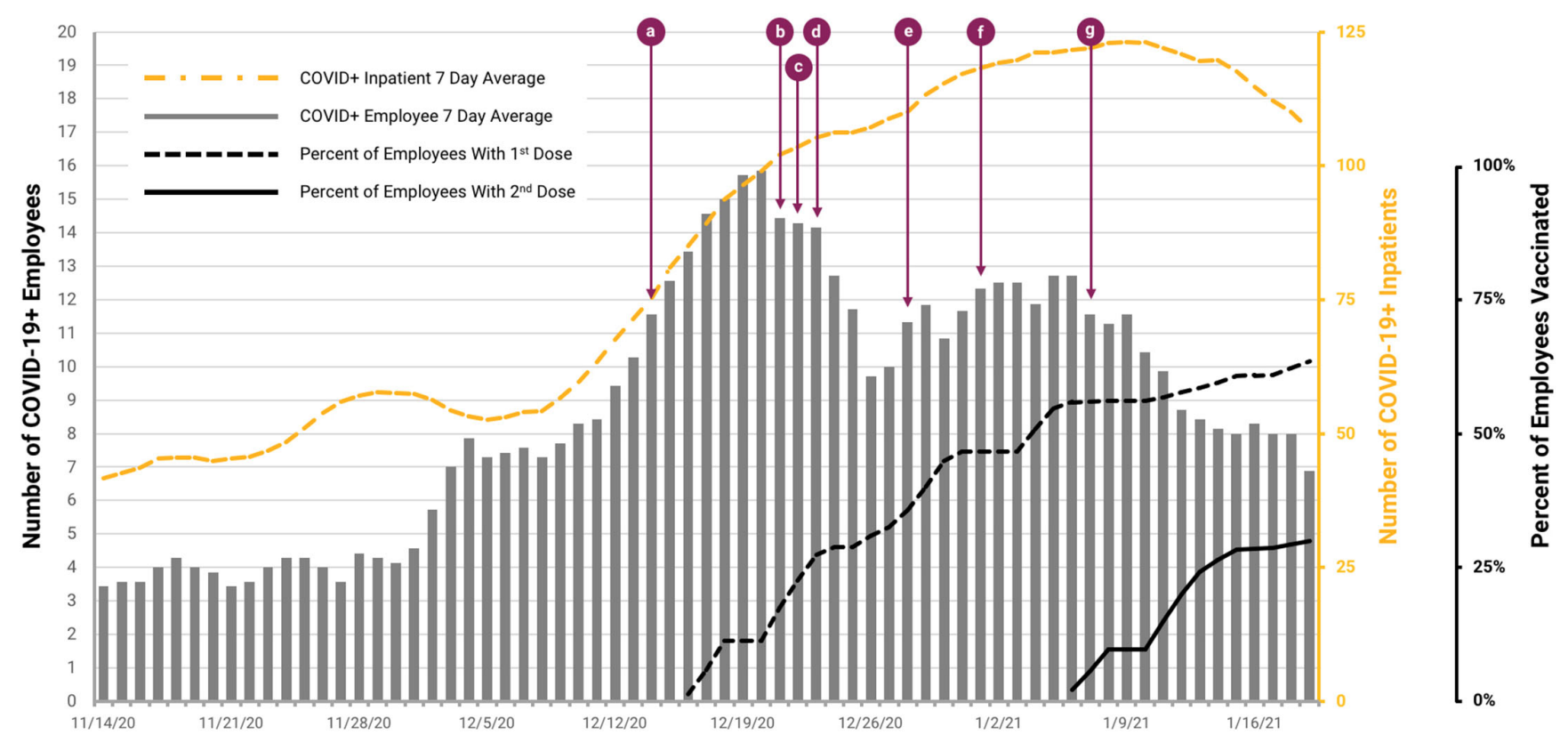

Fig. 1. Bar graph of 7-day average number of health system employees with positive SARS-CoV-2 tests from November 14, 2020, to January 19, 2021. Yellow dashed line is 7-day average number of COVID-19-positive inpatients over the same period. The black dashed line represents the percentage of total health system employees who received first dose of Pfizer anti-SARS-CoV-2 vaccine, beginning December 16, 2020. The black solid line represents the percentage of total health system employees who received second dose of Pfizer anti-SARS-CoV-2 vaccine, beginning January 6, 2021. Other interventions are as follows: (a) December 14, 2020: reduced adult visitors from 2 to 1; (b) December 21, 2020: required face shields and surgical mask for all patient encounters and restricted cafeteria seating; (c) December 22, 2020: employees to confirm symptom free upon arrival; (d) December 23, 2020: employees to use surgical masks in all areas; (e) December 28, 2020: employee temperature screening on arrival; (f) January 1, 2021: 50\% ambulatory visits changes to telehealth; and (g) January 8, 2021: full adult visitor restriction.

2. Polack FP, Thomas SJ, Kitchin N, et al. Safety and efficacy of the BNT162b2 mRNA COVID-19 vaccine. N Engl J Med 2020;383:2603-2615.

3. Baden LR, El Sahly HM, Essink B, et al. Efficacy and safety of the mRNA-1273 SARS-CoV-2 vaccine. N Engl J Med 2020. doi: 10.1056/NEJMoa2035389.

4. Infection control guidance for healthcare professionals about coronavirus (COVID-19). Centers for Disease Control and Prevention website. https://www.cdc.gov/coronavirus/2019-ncov/hcp/infection-control.html. Published 2020. Accessed February 8, 2021.

5. Wood S, Schulman K. Beyond politics-promoting COVID-19 vaccination in the United States. N Engl J Med 2021. doi: 10.1056/NEJMms2033790.

\section{Level of respiratory protection for healthcare workers caring for coronavirus disease 2019 (COVID-19) patients: A survey of hospital epidemiologists}

Winston L. McCormick BS ${ }^{1}$ (D), Michael P. Koster MD ${ }^{1,2}$, Geetika N. Sood MD, ScM ${ }^{3}$ and Leonard A. Mermel DO, ScM ${ }^{1,2}$ ${ }^{1}$ Warren Alpert Medical School of Brown University, Providence, Rhode Island, ${ }^{2}$ Division of Infectious Diseases, Department of Epidemiology \& Infection Control, Rhode Island Hospital, Providence, Rhode Island and ${ }^{3}$ Division of Infectious Diseases, Department of Epidemiology \& Infection Control, Johns Hopkins Bayview Medical Center, Baltimore, Maryland

To the Editor-Appropriate personal protective equipment (PPE) for healthcare workers (HCWs) caring for patients with coronavirus disease 2019 (COVID-19) has not yet been completely

\footnotetext{
Author for correspondence: Dr Leonard Mermel, E-mail: lmermel@lifespan.org Cite this article: McCormick WL, et al. (2022). Level of respiratory protection for healthcare workers caring for coronavirus disease 2019 (COVID-19) patients: A survey of hospital epidemiologists. Infection Control \& Hospital Epidemiology, 43: 681-683, https://doi.org/10.1017/ice.2021.74
}

elucidated nor universally standardized. In areas where severe acute respiratory coronavirus virus 2 (SARS-CoV-2) community transmission is moderate or substantial, the Centers for Disease Control and Prevention (CDC) recommends at least N95 respirator protection, eye protection, gowns, and gloves for patient-facing HCWs. The lack of a centralized national process for the acquisition and stockpiling of PPE has resulted in severe shortages across the United States, forcing competition among hospital systems and leading to extreme measures to procure PPE for HCWs. ${ }^{1,9}$

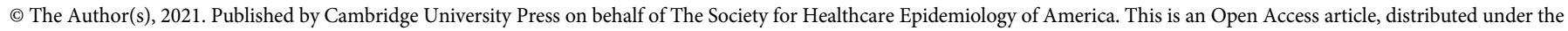

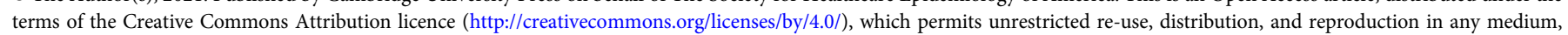
provided the original work is properly cited. 